\title{
A Catalogue of 59,732 Human-Specific Regulatory Sequences Reveals Unique-to-Human Regulatory Patterns Associated with Virus-Interacting Proteins, Pluripotency, and Brain Development
}

\author{
Gennadi V. Glinsky
}

\begin{abstract}
Extensive searches for genomic regions harboring various types of candidate human-specific regulatory sequences (HSRS) identified thousands' HSRS using high-resolution next-generation sequencing technologies and methodologically diverse comparative analyses of human and nonhuman primates' (NHPs) reference genomes. In this study, a comprehensive catalogue of 59,732 genomic loci harboring candidate HSRS has been assembled to facilitate the systematic analyses of genomic sequences that were either inherited from extinct common ancestors (ECAs) or created de novo in human genomes. These analyses identified thousands of candidate HSRS and HSRS-harboring loci that appear inherited from ECAs, yet absent in genomes of our closest evolutionary relatives, chimpanzee and bonobo, presumably due to the incomplete lineage sorting and/or species-specific loss or regulatory DNA. This pattern is particularly prominent for HSRS-harboring loci that have been putatively associated with human-specific gene expression changes in cerebral organoid models. A prominent majority of regions harboring human-specific mutations associated with human-specific expression changes during brain development is highly conserved in chimpanzee, bonobo, and gorilla genomes. Among NHPs, dominant fractions of HSRS-harboring loci associated with human-specific gene expression in both excitatory neurons (347 loci; 67\%) and radial glia (683 loci; 72\%) are highly conserved in the gorilla genome. Analysis of 4433 genes encoding virus-interacting proteins (VIPs) revealed that $95.9 \%$ of human VIPs are components of human-specific regulatory networks that appear to operate in distinct types of human cells from preimplantation embryos to adult dorsolateral prefrontal cortex. These analyses demonstrate that modern humans captured unique genome-wide combinations of regulatory sequences, divergent subsets of which are highly conserved in distinct species of six NHP separated by 30 million years of evolution. Concurrently, this unique-to-human mosaic of genomic regulatory patterns inherited from ECAs was supplemented with 12,486 created de novo HSRS. Genes encoding VIPs appear to represent a principal genomic target during evolution of human-specific regulatory networks, which contribute to fitness of Homo sapiens and affect a functionally diverse spectrum of biological and cellular processes controlled by VIP-containing liquid-liquid phaseseparated condensates.
\end{abstract}

Keywords: human-specific regulatory sequences, human-specific mutations, PtERV1 retrovirus, virus-interacting proteins, human-specific transcription factor binding sites, exaptation of ancestral regulatory DNA

\section{Introduction}

$\mathbf{R}$ ECENT ADVANCES ENABLED by the analyses of individual genomes of great apes using high-resolution sequencing technologies and methodologically diverse comparative analyses of human and nonhuman primates' (NHPs) reference genomes significantly enhanced our understanding of human-specific structural genomic variations of potential regulatory and functional significance (Chimpanzee Sequencing and Analysis Consortium, 2005; Locke et al., 2011; McLean et al., 2011; Konopka et al., 2012; Prüfer et al., 2012; Scally et al., 2012; Shulha et al., 2012; Capra et al., 2013; Marchetto et al., 2013; Marnetto et al., 2014; Gittelman et al., 2015; Prescott et al., 2015; Dong et al., 2016; Dennis et al., 2017; Sousa et al., 2017; Guffanti et al., 2018; Kronenberg et al., 2018; Glinsky et al., 2015, 2016a-c, 2017, 2018). Previous reports were focused on analyses of subsets of loci selected from this expanding genomic universe of human-specific structural variations to highlight mechanistically distinct pathways of their

Institute of Engineering in Medicine, University of California, San Diego, La Jolla, California. 
evolutionary origins (Glinsky, 2016b) (18,364 loci analyzed) or potential impacts on evolution of human-specific traits (reviewed in Levchenko et al., 2018; 4428 loci analyzed). Collectively, these studies markedly expanded the compendium of candidate human-specific regulatory sequences (HSRS), which currently comprises nearly 60,000 genomic loci aligned to the most recent release of the human reference genome (Tables 1-3 and Supplementary Tables S1-S19). This remarkable progress creates a multitude of significant contemporary challenges, the centerpiece of which is a need to compile a comprehensive catalog of HSRS to facilitate identification of the high-priority panel of genetic targets for stringent functional validation experiments. One of the important considerations during the selection of high-priority genetic targets for targeted functional experiments should be focused on illuminating the most compelling evidence linking HSRS and biological pathways that contributed to phenotypic divergence of humans and nonhuman great apes. This approach might help to identify the elite set of HSRS, selection of which will be based on the expectation of high likelihood of biologically significant species-specific effects on phenotypes contributing to segregation of species. Then, in-depth structural-functional explorations of the impact of HSRS on development of humanspecific traits should become feasible. One of essential steps toward addressing these problems is to gain insights into evolutionary origins of genomic regions harboring HSRS and to identify HSRS that are absent in genomes of NHPs.

It has been suggested that regulatory networks associated with the pluripotency phenotype underwent human-specific changes during evolution (Marchetto et al., 2013; Blake et al., 2018). Emergence of human-specific changes of pluripotency regulatory networks operating during human preimplantation embryogenesis was associated with activities of HSRS derived from various families of transposable elements (TE), including stem cell-associated retroviral sequences (Glinsky, 2016a,c, 2017, 2018; Glinsky and Barakat, 2019). These TE-derived regulatory loci include many thousands of transcription factor binding sites (TFBS) and functional enhancer elements (Glinsky, 2016a-c, 2017, 2019; Glinsky and Barakat, 2019), a large fraction of which manifests distinct activities in the adult human brain during physiological and pathological conditions (Guffanti et al., 2018). Expression of several 100,000s of TE-encoded RNA molecules in postmortem samples of the adult human dorsolateral prefrontal cortex (DLPFC) has been documented (Guffanti et al., 2018), more than 4000s of which represent candidate HSRS. Most recently, human-specific gene expression changes in various cell types of developing human and chimpanzee brains have been linked to regulatory loci harboring human-specific mutations (Kronenberg et al., 2018). Collectively, these reports are consistent with the concept that human-specific features of pluripotency regulatory networks highlight a common regulatory nexus of pluripotency with fetal and adult brain development and functions (Glinsky, 2017, 2018), according to which functional priming of HSRS and design of the timeline of their developmental activity maps occur during human preimplantation embryogenesis. Thus, it was of interest to analyze the validity of these observations within the context of a genome-wide global network of nearly 60,000 HSRS.
Consistent with the hypothesis that viruses may represent a major driver of the human lineage adaptation, it has been estimated that interactions with viruses accounted for $\sim 30 \%$ of protein adaptation in the human lineage (Enard and Petrov, 2018; Enard et al., 2016). The diversity, abundance, and biological properties of virus-interacting proteins (VIPs) that are available for engagements during the hostvirus encounter would ultimately determine the susceptibility to infection, and its progression and resolution. However, the biological significance of VIPs is not limited to the sensing and management of host-virus interactions. VIPs represent one of the ubiquitous constituents of membraneless organelles designated liquid-liquid phaseseparated condensates, formation of which is mediated by cooperative interactions of multivalent proteins and nucleic acid molecules (Brangwynne et al., 2009; Banjade et al., 2015; Bergeron-Sandoval et al., 2016; Banani et al., 2017). These structures were originally defined as nuclear or cytoplasmic nonmembrane-bound structures that can be observed as independent domains using transmission electron microscopy without the requirement for antibody labeling for their visualization (Spector, 2006), and now have been functionally implicated in a wide range of biological and cellular processes (see Discussion section). Hence, VIPencoding genes should be considered one of the major targets during the evolution of species-specific genomic regulatory networks. Consequently, it was of interest to determine whether genes encoding VIPs represent the putative downstream regulatory targets of HSRS and to assess what the potential biological effects and significance of these interactions are.

In this contribution, an up-to-date catalog of 59,732 candidate human-specific regulatory loci has been assembled and their conservation patterns in genomes of 5 nonhuman great apes (chimpanzee, bonobo, gorilla, orangutan, and gibbon; Tables 1-3 and Supplementary Table S1-S19) have been analyzed. Systematic comparisons of genomic regions harboring HSRS and unique to African great apes insertions of the PtERV1 retrovirus-derived sequences were carried out resulting in identification of 248 insertions sites of PtERV1 in great apes $(45.9 \% ; p=1.03 \mathrm{E}-44)$ intersecting genomic regions harboring 442 HSRS in humans. Diverse patterns of sequence conservation of different classes of HSRS and HSRS-harboring loci were observed, reflecting quantitatively distinct profiles of inheritance from extinct common ancestors (ECAs) of the human lineage and each of the five species of nonhuman great apes. One of the prevalent modes of evolutionary inheritance is exemplified by thousands of HSRS that appear inherited from ECAs, yet absent in genomes of our closest evolutionary relatives, chimpanzee and bonobo. Significant fractions of retrotransposon-derived loci transcriptionally active in human DLPFC are highly conserved in genomes of gorilla, orangutan, gibbon, and rhesus $(1688 ; 1371 ; 1148$; and 1045 loci, respectively), yet they are absent in genomes of both chimpanzee and bonobo. This mode of evolutionary inheritance was demonstrated for HSRS and HSRS-harboring loci putatively associated with genes implicated in development and functions of human brain. Significantly, these analyses revealed that $95.9 \%$ of human genes encoding VIPs represent apparent targets of human-specific regulatory networks in distinct types of human cells from preimplantation 


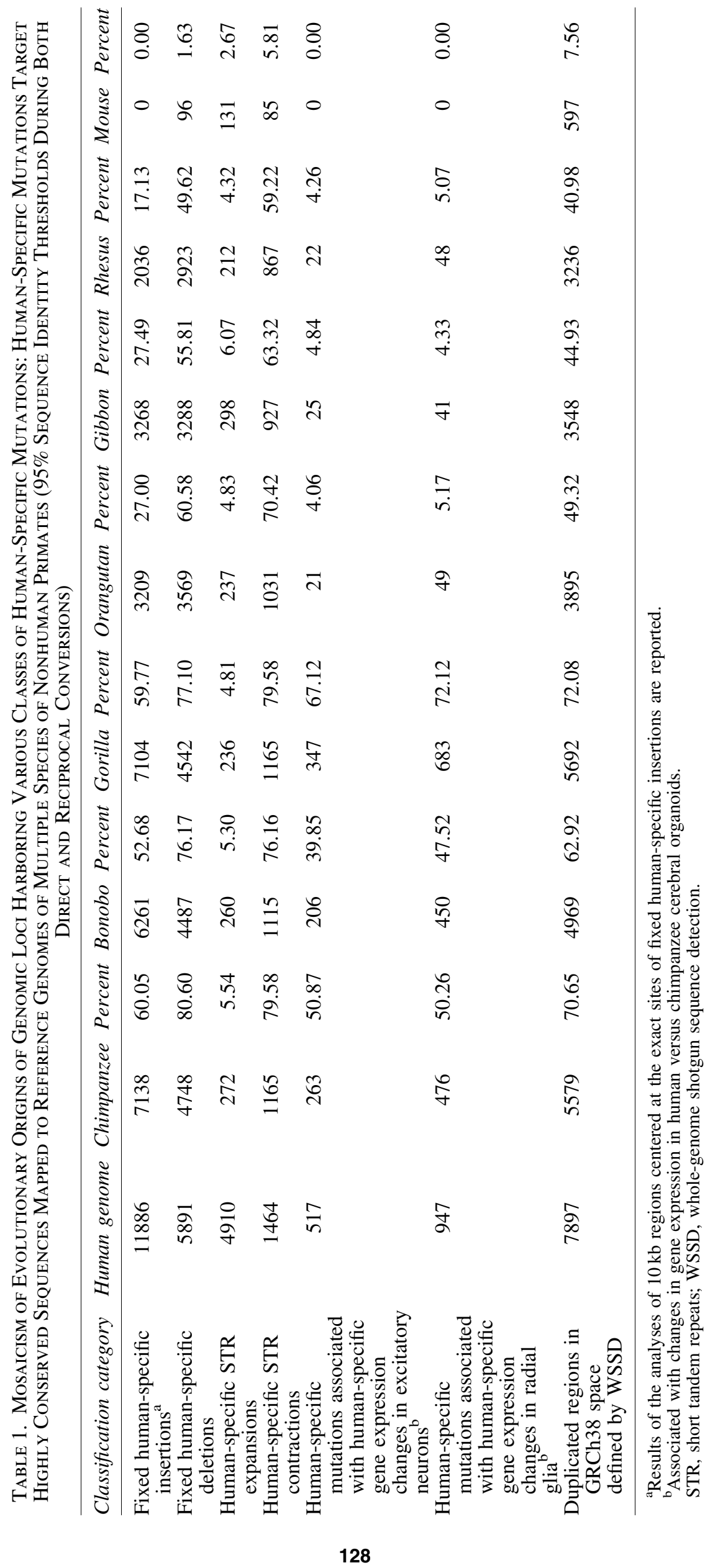




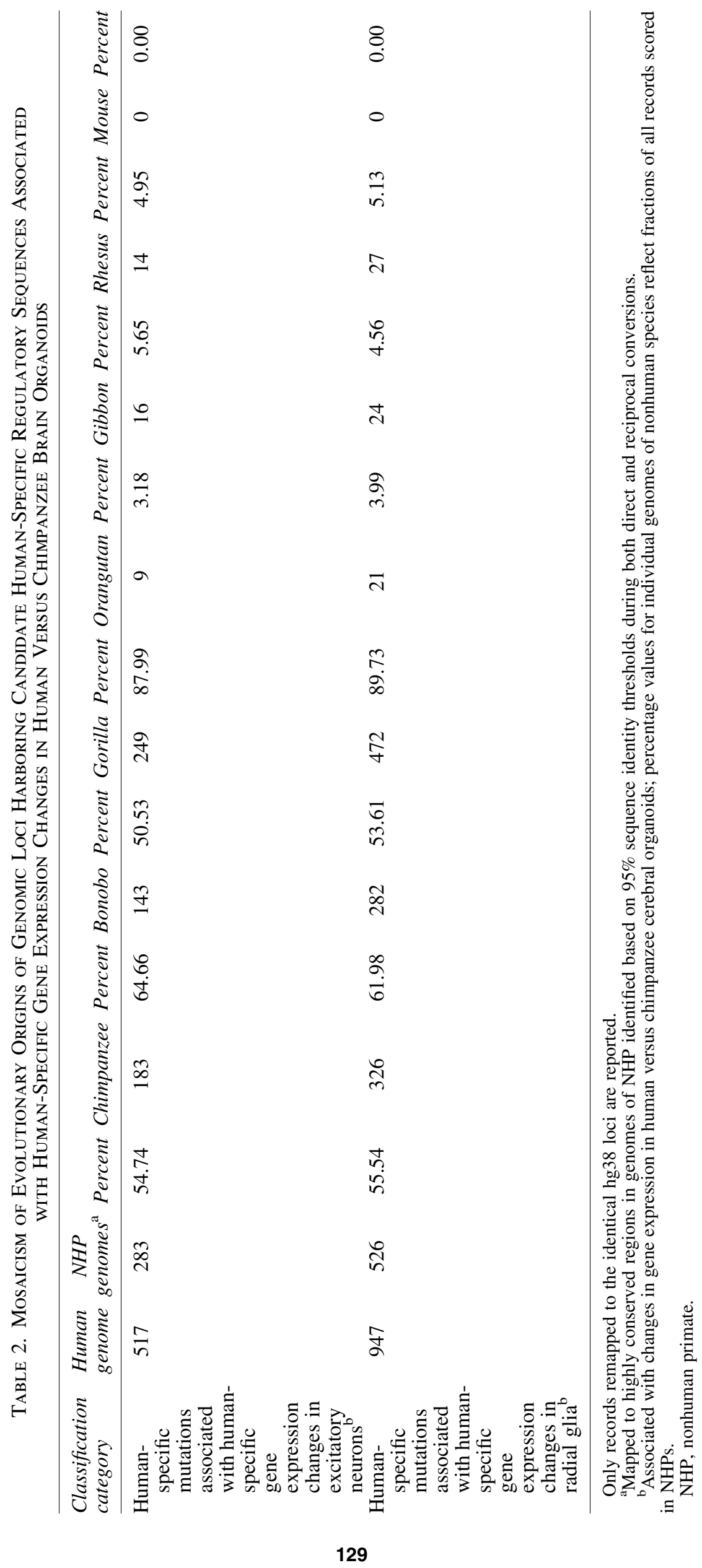


Table 3. Associations of Virus-Interacting Protein-Encoding Genes Comprising Human-Specific Expression Signature of Radial Glia with Human-Specific Mutations

\begin{tabular}{lccccc}
\hline Classification category & VIPs & Non-VIPs & All genes & VIPs, \% & $\mathrm{p}^{\mathrm{a}}$ \\
\hline Up human radial glia & 124 & 259 & 383 & 32.38 & \\
Associated with human-specific mutations & 50 & 16 & 66 & 75.76 & $4.74 \mathrm{E}-11$ \\
Percent & 40.32 & 6.18 & & & \\
$p^{\mathrm{a}}$ & 103 & $1.36 \mathrm{E}-15$ & & & \\
Down human radial glia & 32 & 27 & 285 & 36.14 & \\
Associated with human-specific mutations & 31.07 & 14.84 & 59 & 54.24 & 0.0125 \\
Percent & 227 & 0.0021 & & & \\
$p^{\mathrm{a}}$ & 841 & 668 & 33.98 & \\
All radial glia human-specific genes & 43 & 125 & 65.60 & $6.50 \mathrm{E}-11$ \\
Associated with human-specific mutations & 36.12 & 9.75 & & & \\
Percent & & $9.44 \mathrm{E}-16$ & & & \\
$p^{\mathrm{a}}$ & &
\end{tabular}

${ }^{a} p$-Values were estimated using two-tailed Fisher's exact test.

VIPs, virus-interacting proteins.

embryos to adult DLPFC, suggesting that VIP-encoding genes may represent one of the principal regulatory targets during the evolution and phenotypic divergence of Homo sapiens. Collectively, these observations suggest that manifestations of human-specific phenotypes are controlled by unique-to-human mosaic of human-specific mutations and highly conserved genomic regulatory sequences inherited from ECAs, which is supplemented with 12,486 created de novo HSRS.

\section{Materials and Methods}

\section{Data source}

Candidate HSRS and African ape-specific retroviral insertions. A total of 59,732 candidate HSRS and all currently known 504 insertion sites of the African ape-specific PtERV1 retrovirus (Kronenberg et al., 2018) were analyzed in this study, detailed descriptions of which and corresponding references of primary original contributions are reported in the Tables 1-3 and Supplementary Tables S1-S19.

Additional data sources and analytical protocols. Solely publicly available datasets and resources were used in this contribution as well as methodological approaches and a computational pipeline validated for discovery of primatespecific genes and human-specific regulatory loci (Kent et al., 2002; Schwartz et al., 2003; Tay et al., 2009; Capra et al., 2013; Marnetto et al., 2014; Guffanti et al., 2018; Glinsky, 2015, 2016a-c, 2017, 2018). The analysis is based on the University of California Santa Cruz (UCSC) LiftOver conversion of the coordinates of human blocks to corresponding nonhuman genomes using chain files of precomputed whole-genome BLASTZ alignments with a minMatch of 0.95 and other search parameters in default setting (http://genome.ucsc.edu/cgi-bin/hgLiftOver). Extraction of BLASTZ alignments by the LiftOver algorithm for a human query generates a LiftOver output "Deleted in new," which indicates that a human sequence does not intersect with any chains in a given nonhuman genome. This indicates the absence of the query sequence in the subject genome and was used to infer the presence or absence of the human sequence in the nonhuman reference genome. HSRS were manually curated to validate their identities and genomic features using a BLAST algorithm and the latest releases of the corresponding reference genome databases for time periods between April, 2013, and September, 2018.

The significance of the differences in the expected and observed numbers of events was calculated using two-tailed Fisher's exact test. Additional placement enrichment tests were performed for individual classes of HSRS, taking into account the size in base pairs of corresponding genomic regions. Datasets of NANOG-, POU5F1-, and CTCFbinding sites and human-specific TFBS in hESCs, as well as all other classes of HSRS were reported previously (Kunarso et al., 2010; McLean et al., 2011; Konopka et al., 2012; Prüfer et al., 2012; Scally et al., 2012; Shulha et al., 2012; Capra et al., 2013; Marchetto et al., 2013; Marnetto et al., 2014; Gittelman et al., 2015; Prescott et al., 2015; Dong et al., 2016; Dennis et al., 2017; Sousa et al., 2017; Guffanti et al., 2018; Kronenberg et al., 2018; Glinsky et al., 2015, 2016a-c, 2017, 2018) and are publicly available.

\section{Data analysis}

Categories of DNA sequence conservation. Identification of highly conserved in primates (pan-primate), primatespecific, and human-specific sequences was performed as previously described (Glinsky, 2015, 2016a-c, 2017, 2018). In brief, all categories were defined by direct and reciprocal mapping using liftOver (see previous section, Additional data sources and analytical protocols). Specifically,

- Highly conserved in primates' sequences: DNA sequences that have at least $95 \%$ of bases remapped during conversion from/to human (H. sapiens, hg38), chimp (Pan troglodytes, v5), and bonobo (Pan paniscus, v2, in specifically designated instances, and Pan paniscus, v1 was utilized for comparisons). Similarly, highly conserved sequences were defined for hg38 and genomes of gorilla, orangutan, gibbon, and rhesus.

- Primate specific: DNA sequences that failed to map to the mouse genome (mm10).

- Human specific: DNA sequences that failed to map at least $10 \%$ of bases from human to both chimpanzee and bonobo. All candidate HSRS identified based on the sequence alignments failures to ge- 
nomes of both chimpanzee and bonobo were subjected to more stringent additional analyses requiring the mapping failures to genomes of gorilla, orangutan, gibbon, and rhesus. These loci were considered created de novo HSRS.

To infer the putative evolutionary origins, each evolutionary classification was defined independently by running the corresponding analyses on all candidate HSRS representing the specific category. For example, human-rodent conversion identifies sequences that are absent in the mouse genome based on the sequence identity threshold of $10 \%$. Additional comparisons were performed using the same methodology and exactly as stated in the article text and described in details below.

Genome-wide proximity placement analysis. Genomewide proximity placement analysis of distinct genomic features co-localizing with HSRS was carried out as described previously (Glinsky, 2015, 2016a-c, 2017, 2018). Briefly, as a typical example of the analytical protocol, we examined the significance of overlaps between hESC active enhances and human-specific Transcription Factor Binding Sites (hsTFBS) by first identifying all hsTFBS that overlap with any of the genomic regions tested in the ChIP-STARRseq dataset (Barakat et al., 2018; Glinsky et al., 2018). We then calculated the relative frequency of active enhancers overlapping with hsTFBS. To assess the significance of the observed overlap of genomic coordinates, we compared the values recorded for hsTFBS with the expected frequency of active and nonactive enhancers that overlap with all TFBS for NANOG $(15 \%)$ and OCT4 $(25 \%)$ as previously determined (Barakat et al., 2018). Our analyses demonstrate that more than $95 \%$ of hsTFBS co-localized with sequences in the tested regions of the hESC genome.

Inference of the evolutionary origin and functional enrichment analyses. Evolutionary origins of HSRS were inferred from the results of the conservation patterns of 59,732 candidate human-specific regulatory DNA sequences based on the hg38 release of the human reference genome and latest available releases of genomes of six NHPs, namely chimpanzee, bonobo, gorilla, orangutan, gibbon, and rhesus. The conservation analyses was carried out using the LiftOver algorithm and Multiz Alignments of 20 mammals (17 primates) of the UCSC Genome Browser (Kent et al., 2002) on Human Dec. 2013 Assembly (GRCh38/hg38) (http:// genome.ucsc.edu/cgi-bin/hgTracks?db=hg38\&position $=$ chr $1 \%$ 3A90820922-90821071\&hgsid=441235989_eelAivpkubSY2 AxzLhSXKL5ut7TN).

All DNA sequences were converted to most recent releases of the corresponding reference genome databases and were utilized consistently throughout the study to ensure the use of the most precise, accurate, and reproducible genomic DNA sequences available to date. A candidate HSRS was considered conserved if it could be aligned from/to hg38 reference genome and either one or both chimpanzee or bonobo genomes using defined sequence conservation thresholds of the LiftOver algorithm MinMatch function and direct and reciprocal conversions protocols. Similarly, the conservation patterns were evaluated for genomes of other NHP. LiftOver conversion of the coordinates of human blocks to nonhuman genomes using chain files of precomputed whole-genome BLASTZ alignments with a specified MinMatch levels and other search parameters in default setting. Several thresholds of the LiftOver algorithm MinMatch function (minimum ratio of bases that must remap) were utilized to assess the sequence conservation and identify candidate human specific (MinMatch of $0.1 ; 0.95$; 0.99; and 1.00) and conserved in NHP (MinMatch of 0.95 and 1.00) regulatory sequences as previously described (Guffanti et al., 2018; Glinsky, 2015, 2016a-c, 2017, 2018). The net alignments provided by the UCSC Genome Browser were utilized to compare the sequences in the human genome (hg38) with the mouse (mm10), chimpanzee (PanTro5), and latest available releases of bonobo, gorilla, orangutan, gibbon, and rhesus genomes. A given regulatory DNA segment was defined as the highly conserved regulatory sequence when both direct and reciprocal conversions between humans' and NHPs' genomes were observed using the MinMatch sequence alignment threshold of 0.95 , requiring that $95 \%$ of bases must remap during the alignments of the corresponding sequences. A given regulatory DNA segment was defined as the created de novo candidate HSRS when sequence alignments failed to both chimpanzee and bonobo genomes using the specified MinMatch sequence alignment thresholds. More stringently, these requirements were extended to include genomes of gorilla, orangutan, gibbon, and rhesus. Analyses of conservation patterns of 11,866 human-specific insertions have been performed using 11 different window sizes (Supplementary Table S12) centered at the insertion sites previously reported by Kronenberg et al. (2018). Numbers of records that successfully completed direct and reciprocal conversions from/to hg38 and genomes of nonhuman species (six NHPs and mouse) using sequence identity threshold $95 \%$ are reported in the Supplementary Table S12.

The Enrichr API (January 2018 version) (Chen et al., 2013) was used to test genes linked to HSRS of interest for significant enrichment in numerous functional categories. To comply with the web interface, we considered the 1000 genes closest to the tested peaks for enrichments. In all plots (unless stated otherwise), we report the "combined score" calculated by Enrichr, which is a product of the significance estimate and the magnitude of enrichment (combined score $c=\log (p)^{*} z$, where $p$ is the Fisher's exact test $p$-value and $z$ is the $z$-score deviation from the expected rank). When technically feasible, larger sets of genes comprising several 1000 entries were analyzed. Regulatory connectivity maps between HSGRS and coding genes and additional functional enrichment analyses were performed with GREAT (McLean et al., 2010) at default settings.

Statistical analyses of the publicly available datasets. All statistical analyses of the publicly available genomic datasets, including error rate estimates, background and technical noise measurements, and filtering, feature peak calling, feature selection, assignments of genomic coordinates to the corresponding builds of the reference human genome, and data visualization, were performed exactly as reported in the original publications and associated references linked to the corresponding data visualization tracks (http://genome.ucsc .edu). Any modifications or new elements of statistical 
analyses are described in the corresponding sections of the Results. Statistical significance of the Pearson correlation coefficients was determined using GraphPad Prism version 6.00 software. Both nominal and Bonferroni adjusted $p$ values were estimated. The significance of the differences in the numbers of events between the groups was calculated using two-sided Fisher's exact and chi-square test, and the significance of the overlap between the events was determined using the hypergeometric distribution test (Tavazoie et al., 1999).

\section{Results}

Mosaicism of evolutionary origins of genomic loci harboring various classes of human-specific mutations

Recent experiments identified 24,151 genomic regions harboring various classes of human-specific mutations identified based on the comparative analyses of genomes of modern humans and nonhuman great apes (Kronenberg et al., 2018). It was of interest to analyze the sequence conservation patterns of these 24,151 regions in genomes of 6 NHPs, including 5 nonhuman great apes (chimpanzee, bonobo, gorilla, orangutan, and gibbon) and rhesus macaque (Table 1). In these analyses, genomic sequences that manifested at least $95 \%$ of sequence conservations during the direct and reciprocal conversions from/to reference genomes of modern humans (hg38) and corresponding NHP species were considered highly conserved. Within the context of definition of evolutionary origins of genomic regions harboring human-specific mutations, one of the main motivations was the inference that this analytical effort would identify highly conserved DNA sequences that were inherited by the modern humans' lineage from ECAs.

Consistent with a model of the significant contribution of the ECA inheritance, a majority (66-88\%) of genomic loci harboring various classes of human-specific mutations appeared in genomic regions that are highly conserved in genomes of great apes and rhesus (Table 1). Notably, only $13 \%$ of 4910 human-specific short tandem repeat (STR) expansions' regions are conserved. Consistent with the predominantly primates' origins of regulatory regions harboring human-specific mutations, less than $5 \%$ of analyzed sequences appear highly conserved in the mouse genome (Table 1). Two classes of HSRS were mapped within varying fractions of DNA sequences highly conserved in genomes of different species of NHP: DNA loci harboring 49.6-80.6\% of fixed human-specific deletions and $59.2-79.6 \%$ of humanspecific STR contractions were placed within highly conserved genomic regions in genomes of different species of nonhuman great apes and rhesus (Table 1).

Genomes of the ancestral evolutionary branch leading to human and African great apes show the significant increase in duplication activity (Marques-Bonet et al., 2009; Sudmant et al., 2013) and human-specific segmental duplications have been identified among the most promising candidate genetic loci contributing to the evolution of human-specific phenotypes (Fortna et al., 2004; Charrier et al., 2012; Dennis et al., 2012, 2017; Florio et al., 2015; Kronenberg et al., 2018). It was of interest to analyze evolutionary origins of 7987 duplicated genomic regions that were mapped to the most recent hg38 release of the human reference genome using whole-genome shotgun sequence detection algorithm (Kronenberg et al., 2018). Sequence conservation analyses of genomic regions harboring loci duplicated in the human genome revealed that a vast majority of these regions (regions harboring 6826 duplicated loci; $86.4 \%$ ) are highly conserved in genomes of six NHPs (Table 1 and Supplementary Table S4 and Fig. 1). Interestingly, the largest fractions of both all regions successfully remapped from/to hg38 human reference genome (Table 1 and Fig. 1) and species-specific highly conserved regions (Supplementary Table S4 and Fig. 1) were observed in gorilla and chimpanzee genomes, indicating that these two species of nonhuman great apes appear similarly close to modern humans based on conservation patterns of genomic regions harboring duplicated loci in the human genome. These observations clearly demonstrate the species-specific mosaicism of evolutionary origins of genomic regions harboring duplicated loci in the genome of modern humans (Table 1 and Fig. 1 and Supplementary Table S4).

\section{Mosaicism of evolutionary origins of genomic loci harboring human-specific mutations associated with human-specific gene expression changes in excitatory neurons and radial glia}

Overall, the fractions of highly conserved sequences harboring human-specific mutations and assigned to different NHP species seem to reflect the consensus evolutionary order of the NHP genomes' similarity to the genome of modern humans. Notable exceptions from this pattern were identified during the analyses of human-specific mutations associated with human-specific gene expression changes in excitatory neurons and radial glia (Tables 1 and 2). Among NHP species, a majority of genomic regions harboring human-specific mutations associated with human-specific gene expression changes during brain development in both excitatory neurons and radial glia are highly conserved only in genomes of our three closest evolutionary relatives: $50.9 \%$ and $50.3 \%$ in chimpanzee; $39.9 \%$ and $47.5 \%$ in bonobo; and $67.1 \%$ and $72.1 \%$ in gorilla (for excitatory neurons and radial glia, respectively). In contrast, only $\sim 5 \%$ of genomic regions harboring human-specific mutations associated with human-specific gene expression changes detected in cerebral organoids are highly conserved in genomes of orangutan, gibbon, and rhesus. Among nonhuman great apes, most significant fractions of candidate HSRS associated with human-specific gene expression changes in both excitatory neurons (347 loci; 67\%) and radial glia (683 loci; $72 \%$ ) are located in genomic regions highly conserved in the gorilla genome. For highly conserved regions harboring HSRS associated with human-specific gene expression changes in excitatory neurons, differences in conservation profiles between genomes of gorilla, chimpanzee, and bonobo were highly significant as defined by the twotailed Fisher's exact test $(p=1.434 \mathrm{E}-07 ; p=1.574 \mathrm{E}-18$; $p=0.000463)$. For highly conserved regions harboring HSRS associated with human-specific gene expression changes in radial glia, differences in conservation profiles between genomes of gorilla and chimpanzee as well as gorilla and bonobo were highly significant as well $(p=1.623 \mathrm{E}-22$ and $p=7.369 \mathrm{E}-28$, respectively). In contrast, conservation profiles of HSRS associated with human-specific gene 
A

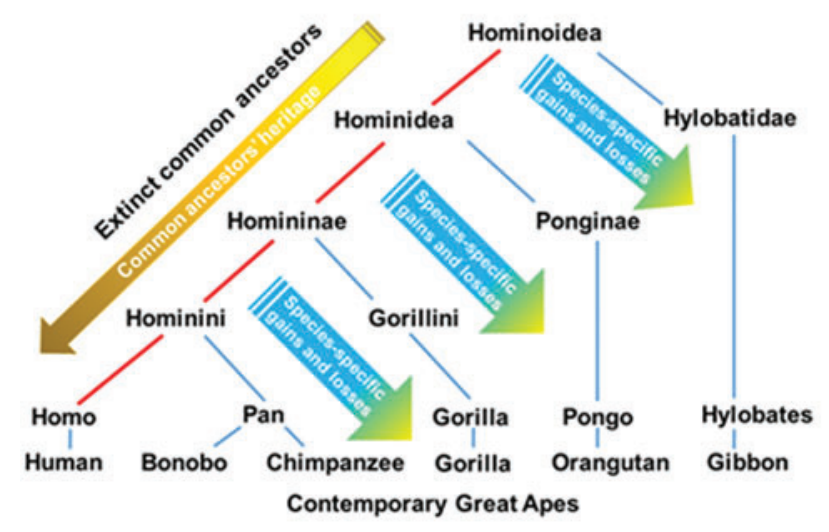

B Mosaicism of evolutionary origins of 7,897 duplicated regions in GRCh38 space defined by WSSD

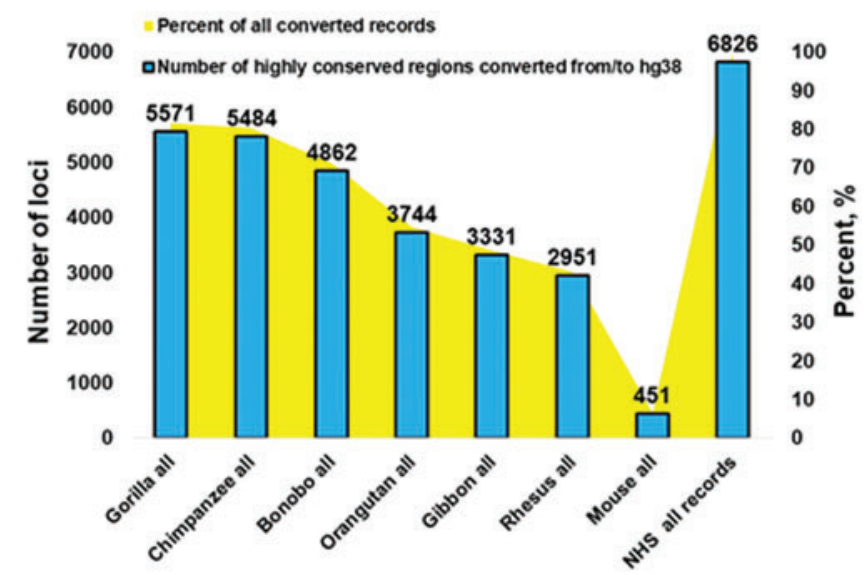

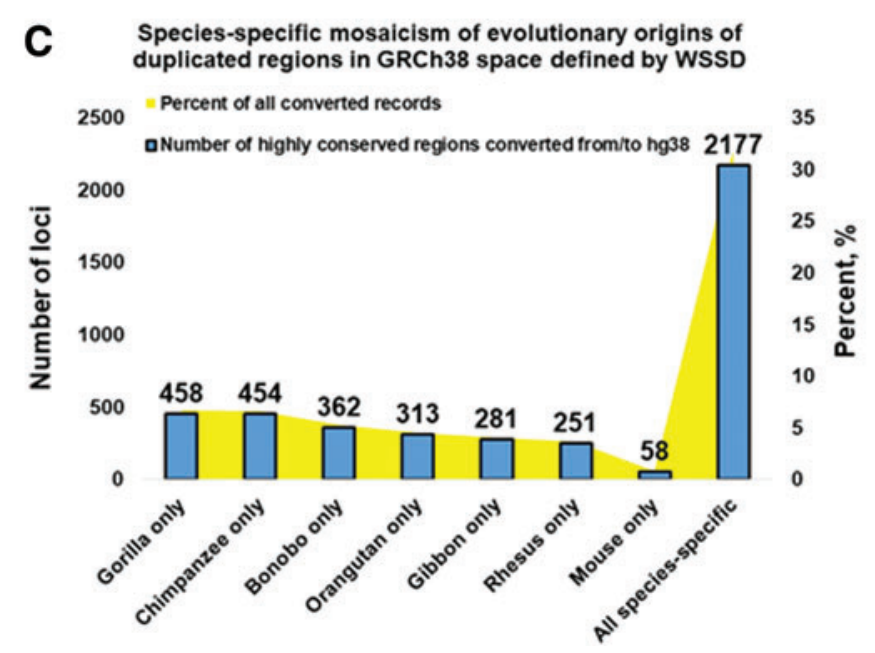

FIG. 1. Mosaicism of evolutionary origins of 7897 duplicated regions in the hg38 release of human reference genome defined by WSSD. (A) A consensus model of the lineage speciation during the evolution of great apes. The arrows depict the hypothetical flow of genomic information inherited from ECAs and acquired through species-specific gain and loss. Evolutionary origins through ECA inheritance pathways of highly conserved sequences in genomes of modern humans and nonhuman species of great apes are postulated. (B) Mosaicism of evolutionary origins of 7897 duplicated regions in the hg38 release of human reference genome defined by WSSD (Kronenberg et al., 2018). Numbers of highly conserved regions that successfully completed direct and reciprocal conversion tests are reported for each NHS. (C) Species-specific mosaicism of evolutionary origins of duplicated regions in the hg38 release of human reference genome defined by WSSD. Only highly conserved sequences identified in the human reference genome and genomes of designated NHS are reported. ECAs, extinct common ancestors; NHS, nonhuman species; WSSD, whole-genome shotgun sequence detection. Color images are available online.

expression changes in radial glia were similar in genomes of chimpanzee and bonobo $(p=0.250$; Table 1$)$. These observations suggest that the majority of highly conserved genomic regions harboring candidate HSRS associated with human-specific differences of gene expression in both excitatory neurons and radial glia was inherited from ECAs of modern humans and gorilla. This conclusion remains valid when the analyses were performed considering either only loci remapped to/from NHP genomes to identical hg38 genomic coordinates (Table 2 and Figs. 2 and 3 and Supplementary Table S4) or only genomic loci uniquely mapped to genomes of only single species of nonhuman great apes (Fig. 3). Notably, differences in conservation profiles of genomic loci harboring HSRS associated with humanspecific gene expression changes in radial glia appear par- ticularly prominent (Table 2 and Figs. 2 and 3 and Supplementary Table S5). Consistent with the ECA inheritance model, from $85.3 \%$ to $95.1 \%$ of HSRS-harboring regions that are highly conserved in the genomes of chimpanzee and bonobo are highly conserved in the gorilla genome as well. In contrast, only from $59.0 \%$ to $59.3 \%$ and from $62.7 \%$ to $65.1 \%$ of HSRS-harboring regions that are highly conserved in the gorilla genome remain highly conserved in the genomes of bonobo and chimpanzee, respectively. These findings were corroborated by observations demonstrating common evolutionary patterns of 248 insertions sites of African ape-specific retrovirus PtERV1 (45.9\%; $p=1.03 \mathrm{E}-$ 44) intersecting genomic regions harboring $442 \mathrm{HSRS}$, which are enriched for HSRS that have been associated with human-specific gene expression changes in cerebral 


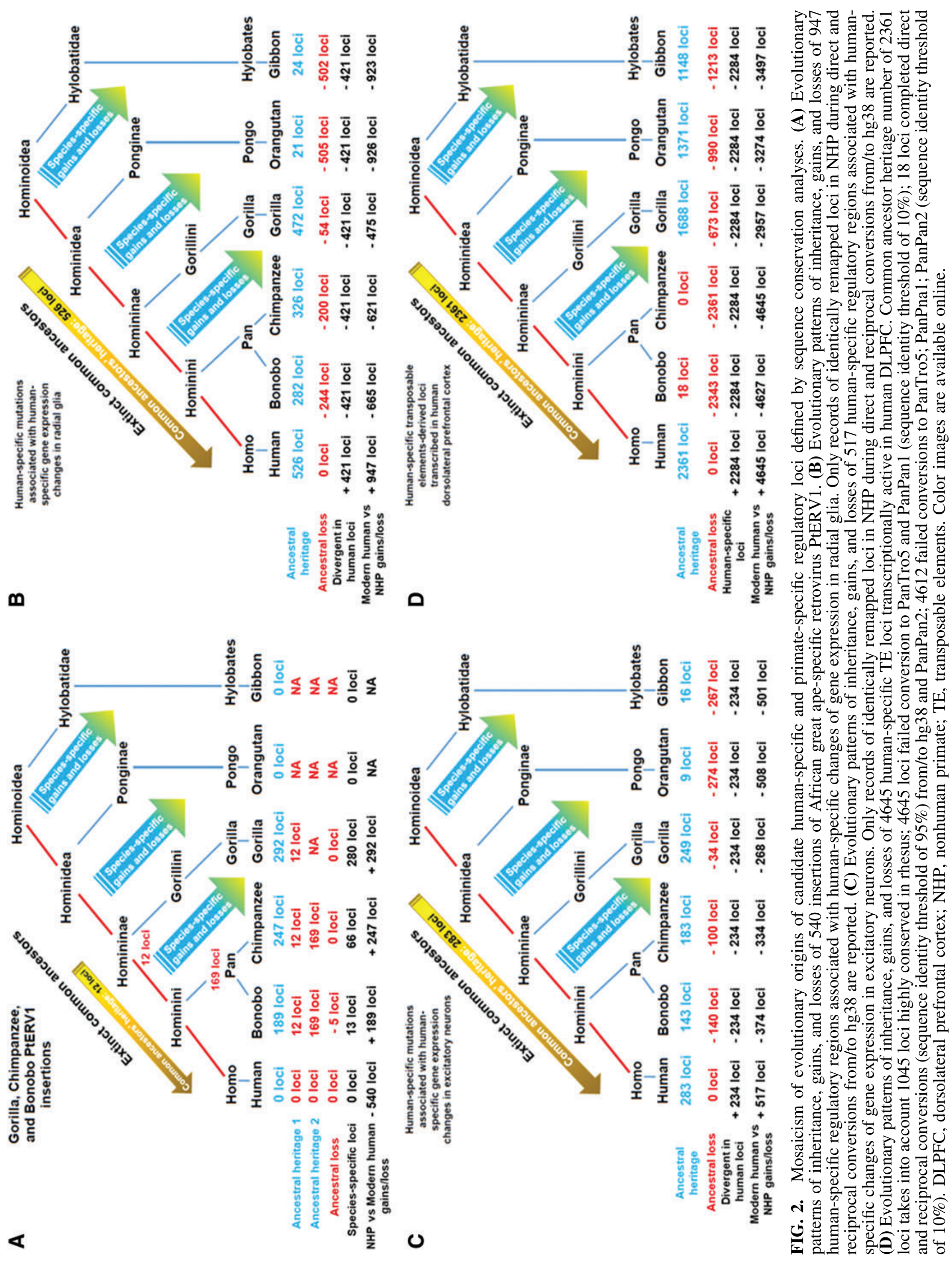




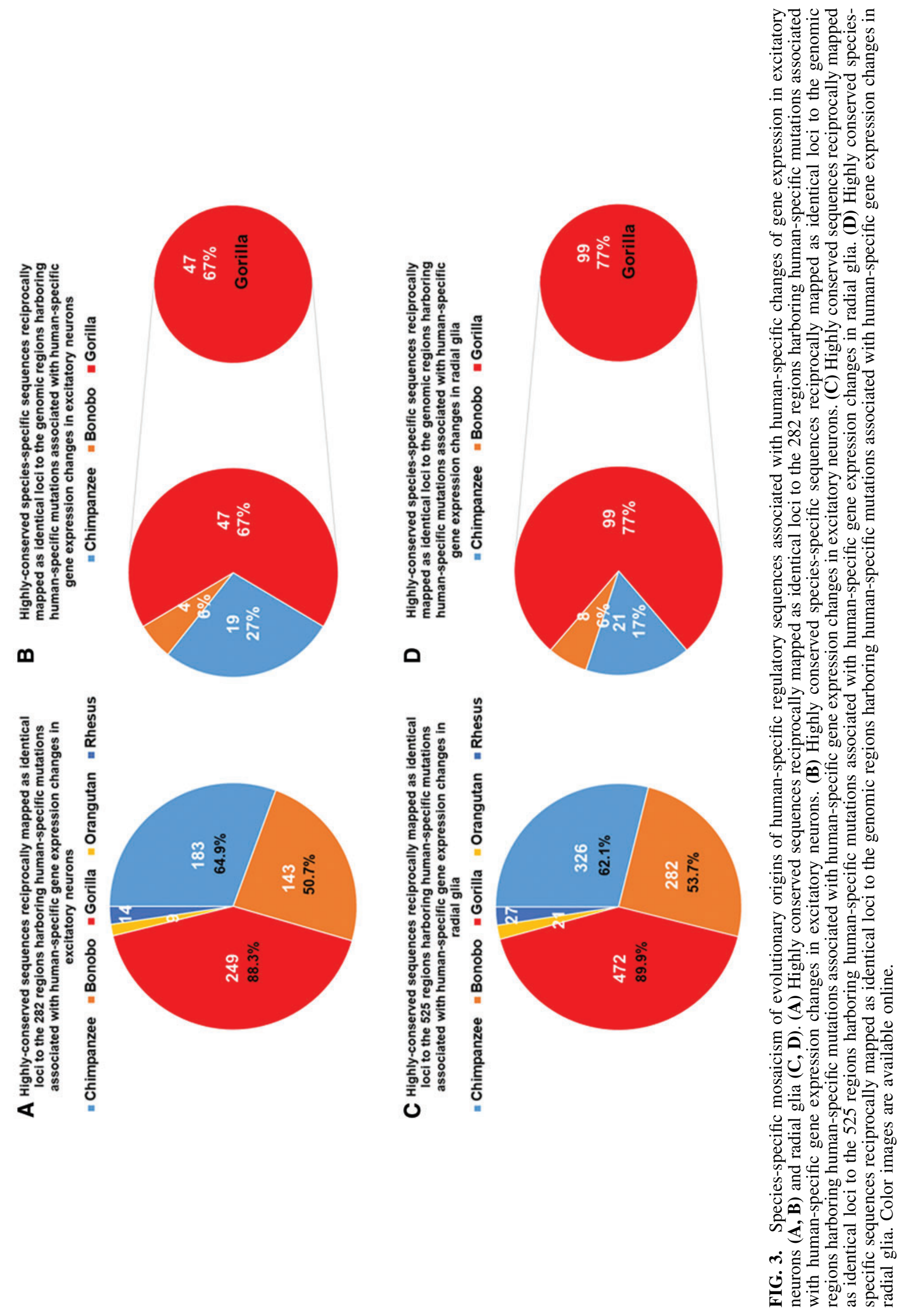


organoid models (Supplementary Data and Supplementary Table S6a-c). Taking into account that placements in defined genomic regions of HSRS and PtERV1 in genomes of human and African apes, respectively, reflect independent stochastic events, these observations are consistent with the hypothesis of potentially significant impact of identified genomic regions on phenotypic divergence of humans and other great apes.

\section{Mosaicism of evolutionary origins of candidate human-specific regulatory loci defined based on the mapping failure to both chimpanzee and bonobo reference genomes}

One of the approaches to the identification of candidate HSRS is based on their absence in the genomes of our closest evolutionary relatives, chimpanzee and bonobo. These analyses demonstrate that large fractions of genomic regions harboring candidate HSRS are highly conserved in genomes of our more distant evolutionary relatives, gorilla, orangutan, gibbon, and rhesus (Tables 1 and 2). These observations suggest that candidate human-specific regulatory loci, which were defined based on the mapping failures to both chimpanzee and bonobo genomes, may originate on DNA sequences highly conserved in genomes of other NHP species. To test the validity of this hypothesis, $16,730 \mathrm{ge}-$ nomic loci harboring distinct classes of HSRS were identified that failed to convert to genomes of both chimpanzee and bonobo using 10\% sequence identity threshold (Supplementary Table S7). Then, highly conserved sequences in genomes of rhesus, gibbon, orangutan, and gorilla were identified and tabulated for each category of HSRS. Consistent with the bypassing patterns of evolutionary inheritance, thousands of distinct classes of candidate HSRS that failed to map to genomes of both chimpanzee and bonobo are highly conserved in genomes of gorilla, orangutan, gibbon, and rhesus (Supplementary Table S7). Most prom- inently, significant fractions of retrotransposon-derived loci that are transcriptionally active in human DLPFC and absent in genomes of both chimpanzee and bonobo are highly conserved in genomes of gorilla, orangutan, gibbon, and rhesus (1688; 1371; 1148; and 1045 loci, respectively; Fig. 4). It has been observed that in all instances, the gorilla genome had the largest numbers of shared with modern humans highly conserved sequences that failed to map to genomes of both chimpanzee and bonobo (Supplementary Table S7).

These observations indicate that a more stringent approach for definition of candidate HSRS, which are likely to have been created de novo in the genome of modern humans, would be to require the conversion failures of a regulatory DNA sequence to all six NHP genomes, namely genomes of chimpanzee, bonobo, gorilla, orangutan, gibbon, and rhesus. Using this strategy, 12,486 candidate HSRS have been identified (Supplementary Table S8), indicating that $24.8 \%$ of all analyzed in this contribution HSRSharboring genomic loci could be classified as created de novo candidate HSRS.

\section{Enrichment within human-specific regulatory pathways of genes comprising expression signatures of human-specific neurodevelopmental and pluripotency transcriptional networks}

It is possible that distinct types of candidate HSRS are not just unrelated elements of a random population of DNA sequences, but they might represent a coherent collection of regulatory DNA sequences assembled during evolution to facilitate execution of human-specific functions. If this hypothesis is correct, then HSRS may represent the key components of human-specific genomic regulatory pathways governing human-specific gene expression patterns observed in various phenotypic contexts associated with humanspecific traits. Therefore, the validity of this hypothesis

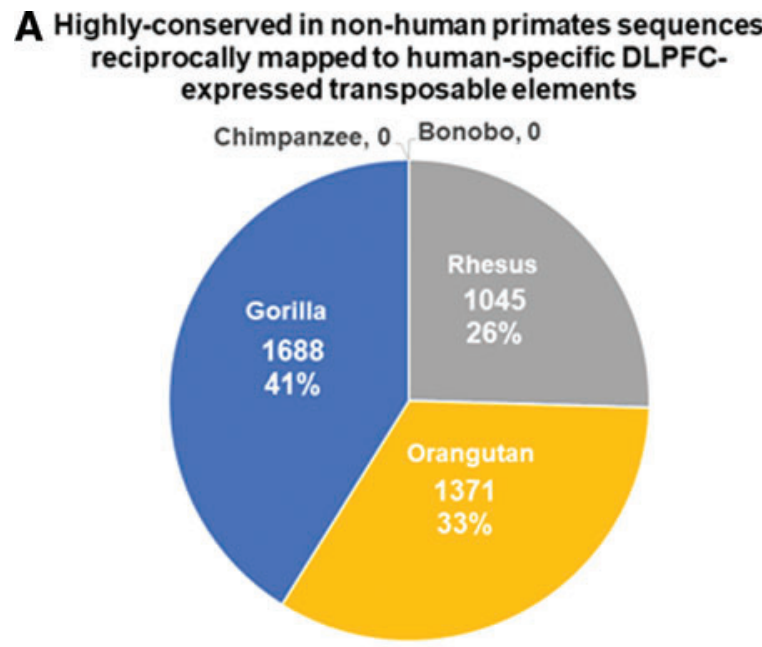

DLPFC, dorsolateral prefrontal cortex

\section{B Highly-conserved species-specific sequences reciprocally mapped to human-specific DLPFC- expressed transposable elements}

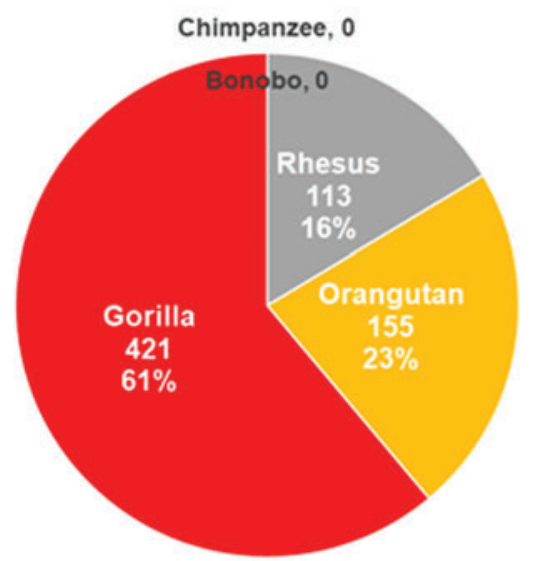

DLPFC, dorsolateral prefrontal cortex

FIG. 4. Species-specific mosaicism of evolutionary origins of human-specific regulatory sequences encoded by transcriptionally active TE in human DPLFC. (A) Numbers of sequences highly conserved in NHP genomes reciprocally mapped to human-specific DLPFC-expressed TE. (B) Highly conserved species-specific sequences reciprocally mapped from NHP genomes to human-specific DLPFC-expressed TE. Color images are available online. 
could be tested using strictly defined by comparisons with nonhuman great apes human-specific gene expression signatures (GESs) associated with development of human brain in the cerebral organoid model (Kronenberg et al., 2018) and induced pluripotency phenotypes of human versus NHP cells (Marchetto et al., 2013). Kronenberg et al. (2018) identified several 100 genes that manifest human-specific expression changes in modern humans versus chimpanzee cerebral organoids' model of brain development. Significant fractions of these human-specific neurodevelopmental networks operating in both excitatory neurons and radial glia appear associated with humanspecific structural variants, specifically with human-specific insertions and deletions (Supplementary Table S9). Even larger fractions of genes comprising human-specific neurodevelopmental networks have been identified as components of the GES of the Multilineage Markers Expressing Cells in human preimplantation embryo: common gene sets represent 262 genes $(68.2 \% ; p=1.59 \mathrm{E}-93)$ and 481 genes $(72.0 \% ; p=3.95 \mathrm{E}-187)$ for human-specific GES of excitatory neurons and radial glia, respectively (Supplementary Table S9).

It has been reported that the creation of the MLME cells in human preimplantation embryos is associated with increased expression of primate-specific retrotransposonderived regulatory long noncoding RNAs termed human pluripotency-associated transcripts (HPATs) (Glinsky et al., 2018). Most recently, the expansive networks of primatespecific and human-specific retrotransposons transcriptionally active in human DLPFC and associated coding genes have been identified (Guffanti et al., 2018). Thus, it was of interest to determine what fractions of genes comprising human-specific neurodevelopmental gene expression networks in excitatory neurons and radial glia may overlap with coding genes coupled with active transcription of TE in human DLPFC. Remarkably, it has been observed that common gene sets represent a vast majority of genes comprising human-specific neurodevelopmental networks: they comprise 322 genes $(83.9 \% ; p=5.57 \mathrm{E}-84)$ and 561 genes $(84 \% ; p=3.08 \mathrm{E}-146)$ for human-specific GES of excitatory neurons and radial glia, respectively (Supplementary Table S9). Interestingly, both SRGAP2C and ARHGAP11B genes driving divergent cortical development between humans and chimpanzee (Charrier et al., 2012; Dennis et al., 2012; Florio et al., 2015) harbor TE transcriptionally active in human DLPFC (Guffanti et al., 2018).

Marchetto et al. (2013) identified human-specific GES distinguishing induced pluripotent stem cells (iPSC) engineered from cells of modern humans and NHP species. To infer the putative regulatory association patterns of genes distinguishing human iPSC versus NHP iPSC and humanspecific genomic regulatory pathways, overlap enrichment analyses of corresponding gene sets have been performed. It has been observed that the association patterns of humanspecific GESs of brain development and pluripotency phenotypes with compendiums of genes likely governed by human-specific regulatory pathways appear strikingly similar (Supplementary Tables S9 and S10). Overall, 88\% of genes comprising human-specific expression signature of the induced pluripotency phenotype represent genes implicated in putative regulatory associations with humanspecific genomic pathways. These observations support the hypothesis that human-specific GESs of brain development and pluripotency phenotypes are associated with a collection of HSRS assembled during evolution into humanspecific genomic regulatory pathways to govern transcriptional networks in human cells.

\section{Digital validation experiments of the enrichment within human-specific regulatory pathways of genes comprising expression signatures of human-specific neurodevelopmental and pluripotency transcriptional networks}

It is important to note that human-specific genomic regulatory networks utilized in these analyses were defined employing vastly different experimental, analytical, and computational approaches that were applied within the broad range of experimental settings: (1) great apes' wholegenome sequencing-guided identification of human-specific insertions and deletions (Kronenberg et al., 2018); (2) genome-wide analysis of retrotransposon's transcriptome in postmortem samples of human DLPFC (Guffanti et al., 2018); (3) shRNA-mediated silencing of LTR7/HERVH retrovirus-derived long noncoding RNAs in human Embryonic Stem Cells (hESC) (Wang et al., 2014); and (4) single-cell expression profiling analyses of human preimplantation embryos (Glinsky et al., 2018). Thus, these human-specific genomic regulatory networks appear to operate in a developmentally diverse spectrum of human cells from preimplantation embryos to adult DLPFC, which collectively represent cell types that are biologically and anatomically highly relevant to manifestations of human-specific phenotypes. To test the validity of the above findings, the independent sets of GESs of humanspecific neurodevelopmental (Supplementary Tables S13S16) (Pollen et al., 2019) and pluripotency (Supplementary Table S17) (Blake et al., 2018) transcriptional networks were analyzed. It has been observed that a total of 817 of 877 (93.2\%) genes comprising genetic markers distinguishing human versus chimpanzee radial glia cells in the cerebral organoids appear associated with human-specific genomic regulatory networks (Supplementary Table S13). Similar association patterns were documented for all other cell types of developing human and chimpanzee brains: common gene sets comprise 708 of 758 (93.4\%) humanspecific marker genes of intermediate progenitors (Supplementary Table S14); 857 of 912 (94\%) human-specific marker genes of excitatory neurons (Supplementary Table S15); and 340 of 383 (88.8\%) human-specific marker genes of inhibitory neurons (Supplementary Table S16). Consistently, 3218 of 3267 (98.5\%) genes distinguishing human versus chimpanzee iPSC overlap with humanspecific genomic regulatory networks operating in human cells (Supplementary Table S17). The enrichment patterns were highly significant for all four analyzed in this study of human-specific genomic regulatory networks (Supplementary Table S13-S17). Based on these observations, it has been concluded that human-specific GESs of brain development and pluripotency phenotypes manifest significant patterns of associations with a collection of HSRS assembled during evolution into human-specific genomic regulatory pathways to govern transcriptional networks in human cells. 
Enrichment within human-specific regulatory pathways of genes encoding VIPS

These analyses identified thousands of genes that are likely regulated by HSRS, among which SERINC5, APO$B E C 3 B$, and PIWIL2 genes are of particular interest because high expression of proteins encoded by these genes is likely to confer increased resistance to the retroviral infection and to restrict the propagation of retrotransposons in human cells (Marchetto et al., 2013; Rosa et al., 2015; Usami et al., 2015). These observations suggest that genes involved in interactions between viruses and human cells may constitute an important category of genetic targets that are placed under control of HSRS during speciation of the human lineage. Therefore, it was of interest to determine whether human genes encoding VIPs are components of humanspecific regulatory networks by analyzing a comprehensive set of more than 4000 genes encoding human VIPs recently reported by Enard and Petrov (2018). The set of VIPencoding genes analyzed in this study comprised 4433 individual records, which were obtained after removal of 3 loci that are no longer in the current ENSEMBL database and adjustment for records that have multiple ENSEMBL identifying numbers associated with the same genomic loci defined by unique gene symbols in hg38 release of the human reference genome database. It has been observed that a significant fraction of genes encoding human VIPs (27.2\%; $p=1.03 \mathrm{E}-66$; the hypergeometric test) appears associated with human-specific structural variants, specifically with human-specific insertions and deletions (Supplementary Table S11). A prominent majority of genes encoding human VIPs have been identified as components of the expression signature of the MLME cells in human preimplantation embryo (3551 VIPs; 80.1\%; Supplementary Table S11) and coding genes coupled with active transcription of
TE in human DLPFC (3574 VIPs; 80.6\%; Supplementary Table S11). Intriguingly, the $L B P 9 / H E R V H$ gene expression pathway regulated in hESC by primate-specific retrovirus LTR7/HERVH-derived long noncoding RNAs appears to control the expression of 2661 VIPs (60\%; Supplementary Table S11). Overall, a vast majority of genes encoding human VIPs (4253 of 4433 genes; 95.9\%) have been identified as putative downstream targets of human-specific genomic regulatory networks.

\section{VIP-encoding genes represent large fractions of genes comprising human-specific expression signatures of radial glia, excitatory neurons, and iPSC}

Above considerations suggest that VIP-encoding genes may be represented among genes comprising humanspecific expression signatures of neurodevelopmental and pluripotency networks (see previous section, Enrichment within human-specific regulatory pathways of genes encoding VIPs). Follow-up analyses revealed that VIPs indeed constitute large fractions of genes comprising humanspecific expression signatures of radial glia $(34 \% ; p=1.51 \mathrm{E}-$ 94; the hypergeometric test; Fig. 5), excitatory neurons (30\%; $p=1.92 \mathrm{E}-41$; Fig. 5); and human iPSC $(25 \% ; p=$ 1.22E-08). Intriguingly, disproportionally large fractions of VIP-encoding genes comprising human-specific expression signatures of both upregulated and downregulated genes in radial glia appear associated with human-specific structural variants (Fig. 5 and Table 3 ). It has been observed that the association with human-specific mutations of VIPs is 6.5fold greater compared to non-VIPs $(p=1.36 \mathrm{E}-15$; twotailed Fisher's exact test) for genes upregulated in human radial glia cells (Fig. 5 and Table 3). For genes downregulated in human radial glia cells (Fig. 5 and Table 3), the
A

VIPs represent large fractions of genes comprising human-specific expression signatures of excitatory neurons ( 384 genes) and radial glia (668 genes) =VIPs $=$ Non-VIPs
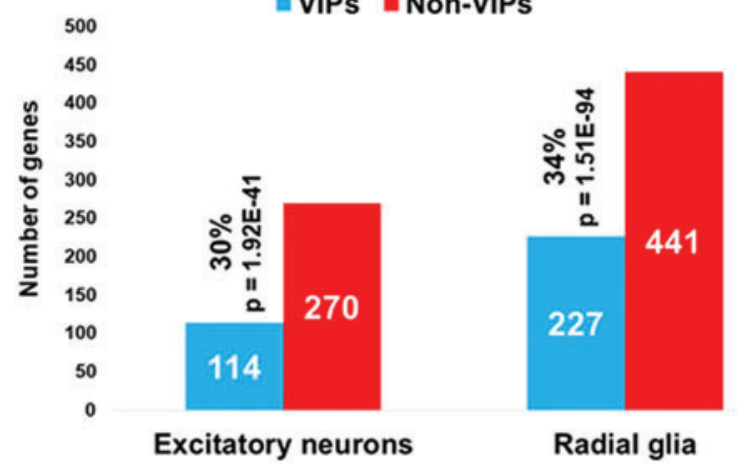
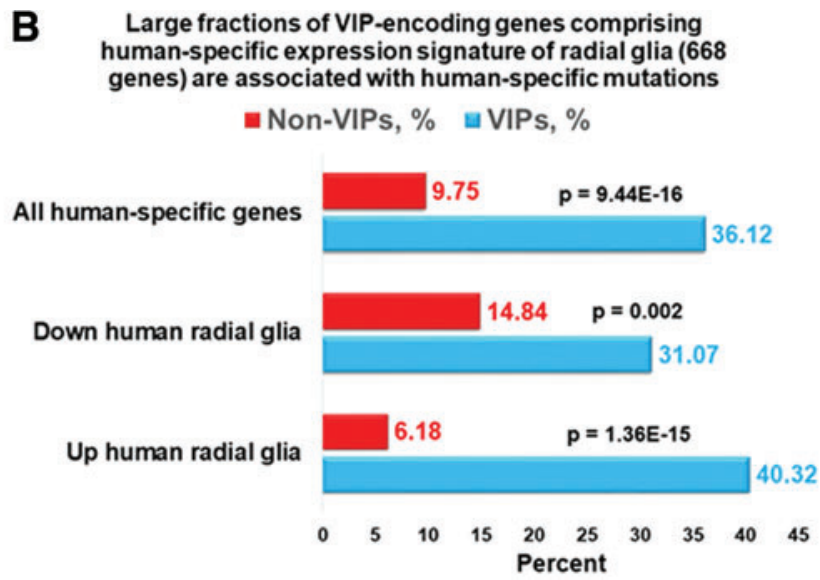

FIG. 5. Genes encoding VIPs represent large fractions of genes comprising human-specific expression signatures of excitatory neurons and radial glia. (A) VIP-encoding genes comprise $34 \%$ and $30 \%$ of genes differentially regulated in human versus chimpanzee radial glia cells and excitatory neurons, respectively, isolated from species-specific cerebral organoids. Significance of the overlaps was estimated using the hypergeometric distribution test. (B) Disproportionally large fractions of both upregulated and downregulated VIP-encoding genes comprising human-specific expression signatures of radial glia cells are associated with human-specific mutations. Corresponding cohorts of genes encoding VIP and non-VIP transcripts associated with human-specific mutations were identified from the set of 668 genes manifesting human-specific expression changes in human versus chimpanzee radial glial cells (Kronenberg et al., 2018). Significance of the differences in associations with human-specific mutations between VIP and non-VIP genes was estimated using the two-tailed Fisher's exact test. VIPs, virus-interacting proteins. Color images are available online. 
association with human-specific mutations of VIPs is 2.1fold greater compared to non-VIPs $(p=0.002$; two-tailed Fisher's exact test). In striking contrast, no increased associations with human-specific mutations have been detected for VIP-encoding genes comprising human-specific expression signatures of excitatory neurons (data not shown), suggesting that observed putatively regulatory associations might be specific to human radial glia cells. Collectively, observations reported in this contribution are highly consistent with the hypothesis that VIP-encoding genes represent important components of human-specific regulatory networks operating in phenotypically distinct human cells that are essential for biological functions of cognition and pluripotency.

\section{Genes encoding VIPs manifest significantly higher enrichment patterns within human-specific genomic regulatory networks compared to non-VIP-encoding genes}

To determine whether larger fractions of genes encoding VIPs are associated with human-specific regulatory networks operating in human cells, we first identified genes associated with each category of HSRS using GREAT algorithm (McLean et al., 2010) and then compiled a set of 13,824 human genes comprising putative regulatory targets associated with HSRS. This set of genes includes 3364 (76\%) VIPencoding genes. Next, the enrichment patterns within humanspecific regulatory networks were independently analyzed for the entire set of 13,824 genes (Supplementary Table S18) and 10,460 non-VIP-encoding genes (Supplementary Table S19). The results were then compared to the enrichment patterns observed for all human genes encoding VIPs (Supplementary Table S11). In all instance, the highest enrichment values were documented for VIP-encoding genes compared to either the entire set of 13,824 genes or the set of 10,460 non-VIPencoding genes $(p<0.0001$; chi-square test with Yates' correction). These observations are consistent with the hypothesis that VIP-encoding genes represent important regulatory targets for HSRS during the evolution of human-specific genomic regulatory networks.

\section{Discussion}

Implications for future structural-functional validation studies of regulatory effects of HSRS

on human-specific regulatory networks

and phenotypic traits

An impressive contemporary collection of nearly 60,000 candidate HSRS assembled by the collective decade-long effort of many laboratories (see Introduction section) lends further credence to the idea that unique-to-human phenotypes might result from human-specific changes to genomic regulatory sequences (King and Wilson, 1975). This study identifies multiple high-priority candidate HSRS for in-depth structural-functional validation analyses, among which most prominent candidate HSRS appears associated with human-specific gene expression changes in excitatory neurons and radial glia as well as in human iPSC. This highpriority set of elite genetic targets include candidate HSRS putatively regulating expression of SERINC5, APOBEC $3 B$, and PIWIL2 genes, high expression of which in human cells is likely to confer increased resistance to the retroviral infection and propagation of retrotransposons (Marchetto et al., 2013; Rosa et al., 2015; Usami et al., 2015). It is tempting to speculate that these changes may have been significant genetic contributors conferring the selective fitness advantage to human lineage during primate evolution.

Observations reported in this contribution have important implications for future functional validation studies, in particular, our approaches to selection and design of experimental systems for follow-up structural-functional analyses of the effects of specific HSRS on defined humanspecific phenotypes. These considerations must take into account the history of evolutionary origins of HSRS to design the specifically tailored experimental models from a panel of cells obtained from modern humans and the appropriate NHP species, genomes of which either have (thus, are appropriate for targeted knock-out and silencing experiments) or not have (thus, are appropriate for knock-in and overexpression experiments) genetic loci of interest. Results of these analyses strongly argue that these experimental systems for targeted interrogation of human-specific genomic regulatory networks should include iPSC as well as brain development models of in vitro induced iPSC differentiation and cerebral organoids. Ideally, these experimental systems should be based on cells obtained from modern humans, our evolutionary closest relatives, chimpanzee and bonobo, as well as gorilla, orangutan, gibbon, and rhesus.

\section{Analyses of evolutionary origins of genomic loci harboring HSRS revealed evidence of a striking ancestral polymorphism and regions of genomic divergence of modern humans and great apes}

High-confidence human-specific mutations leading to emergence of candidate HSRS should be considered rare genomic events that unlikely occur more than once during evolution at the same genomic locations. Therefore, observations that large fractions of genomic regions harboring distinct classes of HSRS represent DNA sequences highly conserved in genomes of distinct NHP species should be interpreted as strong circumstantial evidence consistent with their putative regulatory functions. Collectively, the evidence presented in this contribution revealed a complex unique-to-human mosaic of regulatory DNA sequences inherited from ECAs following separation events from multiple distinct NHP species and reflecting the striking ancestral polymorphism of modern humans. One of the novel mechanisms that may have contributed to divergence of genomic regulatory networks of modern humans and nonhuman great apes is illustrated by observations that the insertions of the African great ape-specific retrovirus PtERV1 and distinct classes of HSRS have common genomic coordinates within orthologous genomic regions of gorilla, chimpanzee, bonobo, and modern humans. Overall, these common patterns of species-specific mutations within overlapping genomic regions were observed for nearly half (248 of 540 loci) of all PtERV1 insertions and 442 HSRS, including 21 HSRS associated with genes differentially expressed in human versus chimpanzee cerebral organoid models of brain development. 
Genomic divergence and speciation of modern humans reflect a complex continuing evolutionary process rather than a singular instantaneous event

Observations reported herein support the hypothesis that the speciation process during evolution of great apes is not likely to occur as an instantaneous event (Patterson et al., 2006): for example, human and chimpanzee lineages could have exchanged genes following the iterative sequences of the initial lineage divergence, separation, and gathering together before the permanent segregation of two species. Since human, chimpanzee, and gorilla lineages may have diverged during the relatively short evolutionary time, this model might reflect the extended complex speciation process of these three closely related great apes, possibly involving co-evolution of their ECAs (Supplementary Fig. S1). Incomplete lineage-sorting events were intrinsic components of the genomic divergence and likely played an important role in the lineage segregation. In agreement with this hypothesis, comparative analyses of multiple alignments of sequences of human, chimpanzee, gorilla, and orangutan genomes have demonstrated that a considerable fraction of genes in the human genome is more similar to the gorilla genome than to the chimpanzee genome (Chen and Li, 2001; O'hUigin et al., 2002; Yang, 2002; Wall, 2003; Patterson et al., 2006; Hobolth et al., 2007). Genomic regions harboring HSRS appear to follow the similar evolutionary trajectory. Observed examples of the bypassing pattern of the evolutionary inheritance highlight HSRS supporting the inference of alternative genealogies (human being most closely related to NHP other than chimpanzee) most likely reflect the incomplete lineage-sorting events. Incomplete lineage sorting has been consistently observed in the multiple alignments of the genomes for human, chimpanzee, gorilla, and orangutan, where differences in models of gene genealogies and species phylogeny were documented for up to $36 \%$ of the human autosomal genome (Chen and Li, 2001; Yang, 2002; Wall, 2003; Patterson et al., 2006; Hobolth et al., 2007, 2011; Kronenberg et al., 2018). Similar changes could result from species-specific losses of conserved ancestral loci of regulatory DNA, a mechanism that contributed to evolution of human-specific traits (McLean et al., 2011).

\section{VIPs as intrinsic protein constituents of liquid-liquid phase-separated condensates contributing to fitness and adaptation of human cells}

These analyses argue that genes encoding VIPs may represent one of the principal genomic elements of humanspecific regulatory networks. However, multiple lines of experimental evidence and theoretical considerations suggest that VIPs may constitute an important regulatory component of the human proteome as well by making a vital contribution to the formation and function of multimolecular complex based on multivalent protein interactions. Intracellular biochemical reactions are spatially compartmentalized within membraneless organelles called cellular bodies, which were originally defined as nuclear or cytoplasmic nonmembrane-bound structures that can be observed as independent domains using transmission electron microscopy without the requirement for antibody labeling for their visualization (Spector, 2006). Typically, these membraneless organelles represent liquid-liquid phase-separated condensates, formation of which is mediated by cooperative interactions of multivalent proteins and nucleic acid molecules (Brangwynne et al., 2009; Banjade et al., 2015; BergeronSandoval et al., 2016; Banani et al., 2017). Cooperative interactions between multivalent proteins and nucleic acid molecules located in close spatial proximity within a cell initiate the formation of liquid-liquid phase-separated condensates appearing as membraneless intracellular organelles, which are being assembled to compartmentalize and accelerate specific biochemical reactions. Most recently, a phase separation model of transcriptional control in mammalian cells has been formulated (Hnisz et al., 2017) and direct experimental evidence supporting the model has been reported (Cho et al., 2018; Sabari et al., 2018).

One of the essential structural features of protein constituents of phase-separated condensates is the propensity for multivalent intermolecular interactions. Notably, it has been reported that human VIPs have more interacting protein partners in the network of human protein-protein interactions than non-VIPs (Stark et al., 2011; Luisi et al., 2015; Enard and Petrov, 2018). These observations indicate that human VIPs may represent intrinsic constituents of many phase-separated membraneless intracellular organelles and these VIP-containing liquid-liquid phaseseparated condensates might be pathogenic targets of viruses. Several independent lines of experimental evidence and theoretical considerations stemming from the concept of intrinsically disordered proteins (IDPs) support this hypothesis.

It has been underscored that IDPs are abundant in the mammalian and viral proteomes (Tarakhovsky and Prinjha, 2018). One of the characteristic features of IDPs is the flexibility of three-dimensional conformations, and this lack of structural constraints of both host and viral IDPs is likely to promote a multiplicity of viral protein interactions with host proteins, thus targeting multiple elements of the host cell defense system (Xue et al., 2014). IDPs are unable to fold spontaneously into stable three-dimensional globular structures and continually fluctuate through a range of conformations (Dyson and Wright, 2005) because they contain high proportions of charged hydrophilic amino acids combined with a relatively low abundance of hydrophobic amino acids. This flexibility of conformational features allows IDPs to form dynamically heterogeneous complexes with multiple binding partners (Ferreon et al., 2009; Ishiyama et al., 2010). The dynamic conformational nature of IDPs is essential for increased exposure and availability of potential binding sites to multiple binding partners, thus facilitating the multivalent intermolecular interactions of IDPs within diverse sets of multiprotein complexes (Tarakhovsky and Prinjha, 2018). Collectively, these features contribute to the apparent governing role of IDPs in signaling networks (Dunker et al., 2005; Dyson and Wright, 2005; Ishiyama et al., 2010). It has been suggested that viral proteins interfere with host phase-separated protein condensates to maximize the impact of viruses on host protein networks and that both viral and host IDPs play a central role in these processes (Tarakhovsky and Prinjha, 2018). It is tempting to speculate that continuing interactions between myriads of viral and host IDPs might represent the major 
force of the evolutionary adaptation on molecular, cellular, and organismal levels.

Taken together, the above considerations strongly argue that human VIPs are components of a large family of IDPs that function as intrinsic protein constituents of many liquidliquid phase-separated multimolecular complexes, facilitating compartmentalization and acceleration of biochemical reactions in human cells. Consistent with this hypothesis, $B R D 4, M E D 1$, and $C D K 9$ genes that were recently identified as the essential structural-functional elements of phaseseparated condensates exerting transcriptional controls at superenhancers (Cho et al., 2018; Sabari et al., 2018) also have been described as VIPs (see database of VIP-encoding genes reported in Enard and Petrov, 2018). Therefore, VIPcontaining phase-separated multimolecular complexes are likely to represent high-value targets for viruses and important fitness determinants during adaptation.

It has been estimated that interactions with viruses accounted for $\sim 30 \%$ of protein adaptation in the human lineage (Enard and Petrov, 2018; Enard et al., 2016), consistent with the hypothesis that viruses appear to be a major driver of adaptation in the human lineage. VIPs evolve under both stronger purifying selection and tend to adapt at much higher rates compared to similar proteins that do not interact with viruses (Enard and Petrov, 2018; Enard et al., 2016). Most recent assessments of the enrichment within VIPs of introgressed segments of genomic DNA after interbreeding of Neanderthals and modern humans provide strong evidence that viruses drive adaptive introgression between species and that adaptive introgression had a substantial impact on VIPs after interbreeding (Enard and Petrov, 2018). This contribution extends the concept of critical importance of VIPs for evolution of modern humans by demonstrating that $95.9 \%$ of all human genes encoding VIPs are components of human-specific genomic regulatory networks that appear to operate in distinct types of human cells ranging from preimplantation embryos to adult DLPFC.

\section{Conclusion}

Observations reported in this contribution support the conclusion that modern humans captured unique combinations of human-specific regulatory loci, divergent subsets of which were created within genomic regions highly conserved in distinct species of six NHP separated by 30 million years of evolution. Concurrently, this unique-to-human mosaic of genomic regulatory pathways built on DNA sequences inherited from ECAs was supplemented with 12,486 created de novo HSRS. Collectively, these findings suggest that incremental genomic divergence of the human lineage has been continued throughout the primate's evolution concurrently with the emergence and segregation of other nonhuman great ape species. This complex continuous process of genomic divergence was gradually driving speciation of $H$. sapiens, in part, by capturing and retaining the unique mosaic of genomic signatures of ECAs. Genes encoding human VIPs are emerging as principal genomic elements of this continuing evolutionary process, contributing to the molecular, cellular, and organismal adaptations by interactions with myriads of viruses.

\section{Acknowledgments}

This work was made possible by the open public access policies of major grant funding agencies and international genomic databases and the willingness of many investigators worldwide to share their primary research data. I would like to thank my anonymous colleagues for their valuable critical contributions during the peer review process of this work.

\section{Author Contributions}

This is a single author contribution. All elements of this work, including the conception of ideas, formulation, and development of concepts, execution of experiments, analysis of data, and writing of the article, were performed by the author.

\section{Disclosure Statement}

No competing financial interests exist.

\section{Funding Information}

No applicable funding.

\section{Supplementary Material}

Supplementary Data

Supplementary Figure S1

Supplementary Table S1

Supplementary Table S2

Supplementary Table S3

Supplementary Table S4

Supplementary Table S5

Supplementary Table S6

Supplementary Table S7

Supplementary Table S8

Supplementary Table S9

Supplementary Table S10

Supplementary Table S11

Supplementary Table S12

Supplementary Table S13

Supplementary Table S14

Supplementary Table S15

Supplementary Table S16

Supplementary Table S17

Supplementary Table S18

Supplementary Table S19

\section{References}

Banani, S.F., Lee, H.O., Hyman, A.A., and Rosen, M.K. (2017). Biomolecular condensates: organizers of cellular biochemistry. Nat Rev Mol Cell Biol 18, 285-298.

Banjade, S., Wu, Q., Mittal, A., Peeples, W.B., Pappu, R.V., and Rosen, M.K. (2015). Conserved interdomain linker promotes phase separation of the multivalent adaptor protein Nck. Proc Natl Acad Sci U S A 112, E6426-E6435.

Barakat, T.S., Halbritter, F., Zhang, M., Rendeiro, A.F., Perenthaler, E., Bock, C., et al. (2018). Functional dissection of the enhancer repertoire in human embryonic stem cells. Cell Stem Cell 23, 276-288.e8. 
Bergeron-Sandoval, L.P., Safaee, N., and Michnick, S.W. (2016). Mechanisms and consequences of macromolecular phase separation. Cell 165, 1067-1079.

Blake, L.E., Thomas, S.M., Blischak, J.D., Hsiao, C.J., Chavarria, C., Myrthil, M., et al. (2018). A comparative study of endoderm differentiation in humans and chimpanzees. Genome Biol 19, 162.

Brangwynne, C.P., Eckmann, C.R., Courson, D.S., Rybarska, A., Hoege, C., Gharakhani, J., et al. (2009). Germline P granules are liquid droplets that localize by controlled dissolution/condensation. Science 324, 1729-1732.

Capra, J.A., Erwin, G.D., McKinsey, G., Rubenstein, J.L., Pollard, K.S. (2013). Many human accelerated regions are developmental enhancers. Philos Trans R Soc Lond B Biol Sci 368, 20130025.

Charrier, C., Joshi, K., Coutinho-Budd, J., Kim, J.E., Lambert, N., de Marchena, J., et al. (2012). Inhibition of SRGAP2 function by its human-specific paralogs induces neoteny during spine maturation. Cell 149, 923-935.

Chen, F.C., and Li, W.H. (2001). Genomic divergences between humans and other hominoids and the effective population size of the common ancestor of humans and chimpanzees. Am J Hum Genet 68, 444-456.

Chen, E.Y., Tan, C.M., Kou, Y., Duan, Q., Wang, Z., Meirelles, G.V., et al. (2013). Enrichr: interactive and collaborative HTML5 gene list enrichment analysis tool. BMC Bioinformatics 14, 128.

Chimpanzee Sequencing and Analysis Consortium. (2005). Initial sequence of the chimpanzee genome and comparison with the human genome. Nature 437, 69-87.

Cho, W.K., Spille, J.H., Hecht, M., Lee, C., Li, C., Grube, V., et al. (2018). Mediator and RNA polymerase II clusters associate in transcription-dependent condensates. Science 361, 412-415.

Dennis, M.Y., Harshman, L., Nelson, B.J., Penn, O., Cantsilieris, S., Huddleston, J., et al. (2017). The evolution and population diversity of human-specific segmental duplications. Nat Ecol Evol 1, 69.

Dennis, M.Y., Nuttle, X., Sudmant, P.H., Antonacci, F., Graves, T.A., Nefedov, M., et al. (2012). Evolution of human-specific neural SRGAP2 genes by incomplete segmental duplication. Cell 149, 912.

Dong, X., Wang, X., Zhang, F., and Tian, W. (2016). Genomewide identification of regulatory sequences undergoing accelerated evolution in the human genome. Mol Biol Evol 33, 2565-2575.

Dunker, A.K., Cortese, M.S., Romero, P., Iakoucheva, L.M., and Uversky, V.N. (2005). Flexible nets. The roles of intrinsic disorder in protein interaction networks. FEBS J 272, 51295148.

Dyson, H.J., and Wright, P.E. (2005). Intrinsically unstructured proteins and their functions. Nat Rev Mol Cell Biol 6, $197-$ 208.

Enard, D., Cai, L., Gwennap, C, and Petrov, D.A. (2016). Viruses are a dominant driver of protein adaptation in mammals. Elife 5, e12469.

Enard, D., and Petrov, D.A. (2018). Evidence that RNA viruses drove adaptive introgression between Neanderthals and Modern Humans. Cell 175, 360-371.

Ferreon, J.C., Martinez-Yamout, M.A., Dyson, H.J., and Wright P.E. (2009). Structural basis for subversion of cellular control mechanisms by the adenoviral E1A oncoprotein. Proc Natl Acad Sci U S A 106, 13260-13265.

Florio, M., Albert, M., Taverna, E., Namba, T., Brandl, H., et al. (2015). Human-specific gene ARHGAP11B promotes basal progenitor amplification and neocortex expansion. Science 347, 1465-1470.

Fortna A., Kim, Y., MacLaren, E., Marshall, K., Hahn, G., et al. (2004). Lineage-specific gene duplication and loss in human and great ape evolution. PLoS Biol 2, e207.

Gittelman, R.M., Hun, E., Ay, F., Madeoy, J., Pennacchio, L., et al. (2015). Comprehensive identification and analysis of human accelerated regulatory DNA. Genome Res 25, 1245-1255.

Glinsky, G.V. (2015). Transposable elements and DNA methylation create in embryonic stem cells human-specific regulatory sequences associated with distal enhancers and non-coding RNAs. Genome Biol Evol 7, 1432-1454.

Glinsky, G.V. (2016a). Activation of endogenous human stem cell-associated retroviruses (SCARs) and therapy-resistant phenotypes of malignant tumors. Cancer Lett 376, 347-359.

Glinsky, G.V. (2016b). Mechanistically distinct pathways of divergent regulatory DNA creation contribute to evolution of human-specific genomic regulatory networks driving phenotypic divergence of Homo sapiens. Genome Biol Evol 8, 2774-2788.

Glinsky, G.V. (2016c). Single cell genomics reveals activation signatures of endogenous SCAR's networks in aneuploid human embryos and clinically intractable malignant tumors. Cancer Lett 381, 176-193.

Glinsky, G.V. (2017). Human-specific features of pluripotency regulatory networks link NANOG with fetal and adult brain development. BioRxiv DOI: https://doi.org/10.1101/022913.

Glinsky, G.V. (2018). Contribution of transposable elements and distal enhancers to evolution of human-specific features of interphase chromatin architecture in embryonic stem cells. Chromosome Res 26, 61-84.

Glinsky, G.V., and Barakat, T.S. (2019). The evolution of Great Apes has shaped the functional enhancers' landscape in human embryonic stem cells. Stem Cell Res 37, 101456.

Glinsky, G., Durruthy-Durruthy, J., Wossidlo, M., Grow, E.J., Weirather, J.L., Au, K.F., et al. (2018). Single cell expression analysis of primate-specific retroviruses-derived HPAT lincRNAs in viable human blastocysts identifies embryonic cells co-expressing genetic markers of multiple lineages. Heliyon 4, e00667.

Guffanti, G., Bartlett, A., Klengel, T., Klengel, C., Hunter, R., Glinsky, G., et al. (2018). Novel bioinformatics approach identifies transcriptional profiles of lineage-specific transposable elements at distinct loci in the human dorsolateral prefrontal cortex. Mol Biol Evol 5, 2435-2453.

Hnisz, D., Shrinivas, K., Young, R.A., Chakraborty, A.K., and Sharp, P.A. (2017). A phase separation model for transcriptional control. Cell 169, 13-23.

Hobolth, A., Christensen, O.F., Mailund, T., and Schierup, M.H. (2007). Genomic relationships and speciation times of human, chimpanzee, and gorilla inferred from a coalescent hidden Markov model. PLoS Genet 3, e7.

Hobolth, A., Dutheil, J.Y., Hawks, J., Schierup, M.H., and Mailund, T. (2011). Incomplete lineage sorting patterns among human, chimpanzee, and orangutan suggest recent orangutan speciation and widespread selection. Genome Res 21, 349-356.

Ishiyama, N., Lee, S.H., Liu, S., Li, G.Y., Smith, M.J., Reichardt, L.F., et al. (2010). Dynamic and static interactions between p120 catenin and E-cadherin regulate the stability of cell-cell adhesion. Cell 141, 117-128.

Kent, W.J., Sugnet, C.W., Furey, T.S., Roskin, K.M., Pringle, T.H., Zahler, A.M., et al. (2002). The human genome browser at UCSC. Genome Res 12, 996-1006. 
King, M.C., and Wilson, A.C. (1975). Evolution at two levels in humans and chimpanzees. Science 188, 107-116.

Konopka, G., Friedrich, T., Davis-Turak, J., Winden, K., Oldham, M.C., Gao, F., et al. (2012). Human-specific transcriptional networks in the brain. Neuron 75, 601-617.

Kronenberg, Z.N., Fiddes, I.T., Gordon, D., Murali, S., Cantsilieris, S., Meyerson, O.S., et al. (2018). High-resolution comparative analysis of great ape genomes. Science 360, eaar6343.

Kunarso, G., Chia, N.Y., Jeyakani, J., Hwang, C., Lu, X., Chan, Y.S., et al. (2010). Transposable elements have rewired the core regulatory network of human embryonic stem cells. Nat Genet 42, 631-634.

Levchenko, A., Kanapin, A., Samsonova, A., and Gainetdinov, R.R. (2018). Human accelerated regions and other humanspecific sequence variations in the context of evolution and their relevance for brain development. Genome Biol Evol 10, 166-188.

Locke, D.P., Hillier, L.W., Warren, W.C., Worley, K.C., Nazareth, L.V., Muzny, D.M., et al. (2011). Comparative and demographic analysis of orangutan genomes. Nature 469, 529-533.

Luisi, P., Alvarez-Ponce, D., Pybus, M., Fares, M.A., Bertranpetit, J., and Laayouni, H. (2015). Recent positive selection has acted on genes encoding proteins with more interactions within the whole human interactome. Genome Biol Evol 7, 1141-1154.

Marchetto, M.C.N., Narvaiza, I., Denli, A.M., Benner, C., Lazzarini, T.A., Nathanson, J.L., et al. (2013). Differential LINE-1 regulation in humans and other great apes. Nature 503, 525-529.

Marnetto, D., Molineris, I., Grassi, E., and Provero, P. (2014). Genome-wide identification and characterization of fixed human-specific regulatory regions. Am J Hum Genet 95, 39-48.

Marques-Bonet, T., Kidd, J.M., Ventura, M., Graves, T.A., Cheng, Z., Hillier, L.W., et al. (2009). A burst of segmental duplications in the genome of the African great ape ancestor. Nature 457, 877-881.

McLean, C.Y., Bristor, D., Hiller, M., Clarke, S.L., Schaar, B.T., Lowe, C.B., et al. (2010). GREAT improves functional interpretation of cis-regulatory regions. Nat Biotechnol 28, 495-501.

McLean, C.Y., Reno, P.L., Pollen, A.A., Bassan, A.I., Capellini, T.D., Guenther, C., et al. (2011). Human-specific loss of regulatory DNA and the evolution of human-specific traits. Nature 471, 216-219.

O'hUigin, C., Satta, Y., Takahata, N., and Klein, J. (2002). Contribution of homoplasy and of ancestral polymorphism to the evolution of genes in anthropoid primates. Mol Biol Evol 19, 1501-1513.

Patterson, N., Richter, D.J., Gnerre, S., Lander, E.S., and Reich, D. (2006). Genetic evidence for complex speciation of human and chimpanzees. Nature 441, 1103-1108.

Pollen, A.A., Bhaduri, A., Andrews, M.G., Nowakowski, T.J., Meyerson, O.S., Mostajo-Radji, M.A., et al. (2019). Establishing cerebral organoids as models of human-specific brain evolution. Cell 176, 743-756.

Prescott, S.L., Srinivasan, R., Marchetto, M.C., Grishina, I., Narvaiza, I., Selleri, L., et al. (2015). Enhancer divergence and cis-regulatory evolution in the human and chimp neural crest. Cell 163, 68-83.

Prüfer, K., Munch, K., Hellmann, I., Akagi, K., Miller, J.R., Walenz, B., et al. (2012). The bonobo genome compared with the chimpanzee and human genomes. Nature 486, 527-531.

Rosa, A., Chande, A., Ziglio, S., De Sanctis, V., Bertorelli, R., Goh, S.L., et al. (2015). HIV-1 Nef promotes infection by excluding SERINC5 from virion incorporation. Nature 526, 212-217.

Sabari, B.R., Dall'Agnese, A., Boija, A., Klein, I.A., Coffey, E.L., Shrinivas, K., et al. (2018). Coactivator condensation at super-enhancers links phase separation and gene control. Science 361, eaar3958.

Scally, A., Dutheil, J.Y., Hillier, L.W., Jordan, G.E., Goodhead, I., Herrero, J., et al. (2012). Insights into hominid evolution from the gorilla genome sequence. Nature 483, 169-175.

Schwartz, S., Kent, W.J., Smit, A., Zhang, Z., Baertsch, R., Hardison, R.C., et al. (2003). Human-mouse alignments with BLASTZ. Genome Res 13, 103-107.

Shulha, H.P., Crisci, J.L., Reshetov, D., Tushir, J.S., Cheung, I., Bharadwaj, R., et al. (2012). Human-specific histone methylation signatures at transcription start sites in prefrontal neurons. PLoS Biol 10, e1001427.

Sousa, A.M.M., Meyer, K.A., Santpere, G., Gulden, F.O., and Sestan, N. (2017). Evolution of the human nervous system function, structure, and development. Cell 170, 226-247.

Spector, D.L. (2006). SnapShot: cellular bodies. Cell 127, 1071. Stark, C., Breitkreutz, B.J., Chatr-Aryamontri, A., Boucher, L., Oughtred, R., Livstone, M.S., et al. (2011). The BioGRID Interaction Database: 2011 update. Nucleic Acids Res 39, D698-D704.

Sudmant, P.H., Huddleston, J., Catacchio, C.R., Malig, M., Hillier, L.W., Baker, C., et al. (2013). Evolution and diversity of copy number variation in the great ape lineage. Genome Res 23, 1373-1382.

Tarakhovsky, A., and Prinjha, R.K. (2018). Drawing on disorder: How viruses use histone mimicry to their advantage. J Exp Med 215, 1777-1787.

Tavazoie, S., Hughes, J.D., Campbell, M.J., Cho, R.J., and Church, GM. (1999). Systematic determination of genetic network architecture. Nat Genet 22, 281-285.

Tay, S.K., Blythe, J., and Lipovich, L. (2009). Global discovery of primate-specific genes in the human genome. Proc Natl Acad Sci U S A 106, 12019-12024.

Usami, Y., Wu, Y., and Göttlinger, H.G. (2015). SERINC3 and SERINC5 restrict HIV-1 infectivity and are counteracted by Nef. Nature 526, 218-223.

Wall, J.D. (2003). Estimating ancestral population sizes and divergence times. Genetics 163, 395-404.

Wang, J., Xie, G., Singh, M., Ghanbarian, A.T., Raskó, T., Szvetnik, A., et al. (2014). Primate-specific endogenous retrovirus-driven transcription defines naive-like stem cells. Nature 516, 405-409.

Xue, B., Blocquel, D., Habchi, J., Uversky, A.V., Kurgan, L., Uversky, V.N., et al. (2014). Structural disorder in viral proteins. Chem Rev 114, 6880-6911.

Yang, Z.H. (2002). Likelihood and Bayes estimation of ancestral population sizes in hominoids using data from multiple loci. Genetics 162, 1811-1823.

Address correspondence to: Gennadi V. Glinsky, MD, PhD Institute of Engineering in Medicine University of California, San Diego 9500 Gilman Drive MC 0435 La Jolla, CA 92093-0435

E-mail: gglinskii@ucsd.edu

Received for publication July 27, 2019; received in revised form August 29, 2019; accepted September 30, 2019. 